\title{
A Chromosomal Gene for Chloramphenicol Acetyltransferase in Streptomyces acrimycini
}

\author{
By HELEN M. WRIGHT AND DAVID A. HOPWOOD \\ John Innes Institute, Colney Lane, Norwich $N_{4}{ }_{7} U H$
}

(Received 25 May 1977)

\section{INTRODUCTION}

Plasmid-borne genes determining antibiotic resistance in eubacteria may have originated in the organisms that produce the antibiotics, notably the streptomycetes (Benveniste \& Davies, 1973; Courvalin, Weisblum \& Davies, 1977). Although this theory does not require the corresponding genes to be permanently plasmid-linked in their streptomycete hosts, the normal carriage of such genes on plasmids might have favoured their dissemination from such hosts to other bacteria. Of the antibiotic-inactivating enzymes coded by eubacterial plasmids, those catalysing acetylation or phosphorylation of aminoglycosides or acetylation of chloramphenicol are found in various streptomycetes (Benveniste \& Davies, 1973; El-Kersch \& Plourde, I976a; Shaw \& Hopwood, 1976; Nakano et al., 1977). However, nothing appears to be known in these organisms about the genetic determination of these enzymes because they have not been studied in genetically characterized strains. We set out to answer this question for a streptomycete producing chloramphenicol acetyltransferase (CAT).

The genetically well-known Streptomyces coelicolor A3(2) is somewhat more resistant to chloramphenicol than most other strains tested, and gives rise to chloramphenicol-sensitive variants at a high frequency, but these do not arise by plasmid loss; nor does absence of CAT explain their phenotype since the parent strain lacks this enzyme (Freeman, Bibb \& Hopwood, 1977). In order to study the genetic determination of CAT in a streptomycete, we therefore had to choose one of three genetically uninvestigated species which possess the enzyme (Shaw \& Hopwood, 1976). We selected Streptomyces acrimycini IPVI610 because of its added interest in yielding a mutant with greatly increased CAT production (Shaw \& Hopwood, 1976); this mutant has allowed the CAT produced by the organism to be compared with those of eubacteria, including those determined by plasmids (Zaidenzaig \& Shaw, 1977).

\section{METHODS}

Media, strains and standard genetical procedures. Complete (CM) and minimal (MM) media and supplementation of MM with growth factors or streptomycin were as described by Hopwood (1967). Soft nutrient agar was Oxoid Nutrient Agar diluted with an equal volume of Oxoid Nutrient Broth. The starting strain was $S$. acrimycini IPVI6Io (John Innes stock no. 1201). Auxotrophic and streptomycin-resistant mutants of this strain, isolated during the work, are referred to in the Results. Escherichia coli was strain J53 of E. coli K12. Incubation of Streptomyces cultures was at $30^{\circ} \mathrm{C}$ and of E. coli at $37^{\circ} \mathrm{C}$. Standard cultural and crossing techniques were those described by Hopwood (1967) and Hopwood et al. (1973) for $S$. coelicolor.

Mutagenesis and mutant isolation. Treatments with $N$-methyl- $N^{\prime}$-nitro- $N$-nitrosoguanidine (NTG) (Delić, Hopwood \& Friend, 1970) and with ultraviolet light (u.v.) (Harold \& Hopwood, 1970) were by standard procedures, as were methods for the isolation of auxotrophic and streptomycin-resistant mutants. Isolation of putative CAT- mutants was attempted by two methods which depended, respectively, on hypotheses that they would, or would not, be more sensitive to chloramphenicol than the starting strain, a proline auxotroph of strain 1201. In the first method, mutagenized spore suspensions were plated on CM to give about 100 colonies per plate. These were replica-plated to MM supplemented with proline and containing $10 \mu \mathrm{g}$ 
chloramphenicol $\mathrm{ml}^{-1}$. Colonies that failed to replicate, representing potential chloramphenicol-sensitive and auxotrophic mutants, were picked to $\mathrm{CM}$ and re-tested by replica-plating to MM supplemented with proline, with and without chloramphenicol. The chloramphenicol-sensitive variants were tested for their ability to inactivate chloramphenicol. They were inoculated (20 per plate) on medium lacking chloramphenicol, a plug ( $7 \mathrm{~mm}$ diam.) was cut from each culture, and the plugs were embedded (20 per dish) in.MM containing Io $\mu \mathrm{g}$ chloramphenicol $\mathrm{ml}^{-1}$. After $24 \mathrm{~h}$ incubation the plates were overlaid with $5 \mathrm{ml}$ soft nutrient agar containing $E$. coli 353 , which was sensitive to chloramphenicol at 2 to $3 \mu \mathrm{g} \mathrm{ml}^{-1}$, and incubated overnight. Each CAT-containing culture was surrounded by a turbid zone in which destruction of chloramphenicol had allowed the growth of $E$. coli. In the second method, colonies developing on CM from mutagenized spore suspensions were picked (50 per plate) to supplemented MM containing $15 \mu \mathrm{g}$ chloramphenicol $\mathrm{ml}^{-1}$ and incubated for 24 to $36 \mathrm{~h}$. The plates were overlaid with $5 \mathrm{ml}$ soft nutrient agar containing $E$. coli and incubated overnight. Each CAT-containing culture was covered by a turbid zone of bacterial growth.

Determination of chloramphenicol acetyltransferase. This was done by the autoradiographic procedure of Shaw \& Hopwood (1976).

\section{RESULTS}

\section{Isolation and characteristics of a $C A T^{-}$mutant}

Since the minimum inhibitory concentration of chloramphenicol in agar for $S$. acrimycini I $20 \mathrm{I}$ is about $10 \mu \mathrm{g} \mathrm{ml}^{-1}$, a concentration tolerated by most of the other wild types tested including those which lacked CAT (Shaw \& Hopwood, 1976), we could not predict whether or not a $\mathrm{CAT}^{-}$mutant would be more sensitive to chloramphenicol than the parent strain. We therefore attempted to isolate such mutants by procedures which depended on their being sensitive, or resistant, respectively to the concentration of the antibiotic tolerated by the parent strain.

In the first method, 775 survivors of u.v. treatment yielded $24(4 \%)$ chloramphenicolsensitive variants and seven (1 \%) auxotrophs. After NTG treatment, 7455 colonies yielded $402(5.4 \%)$ chloramphenicol-sensitive variants and $172(2.3 \%)$ auxotrophs. Out of the 426 chloramphenicol-sensitive variants, one (from the NTG treatment) failed to inactivate chloramphenicol. This single putative $\mathrm{CAT}^{-}$mutant (strain 2498), which retained the proline marker of the parent strain, showed no detectable CAT activity when tested by the autoradiographic method. Its sensitivity to chloramphenicol was similar to that of the majority of chloramphenicol-sensitive but $\mathrm{CAT}^{+}$variants, tolerating $\mathrm{I}$ to $2 \mu \mathrm{g} \mathrm{ml}^{-1}$. The chloramphenicol-sensitive variants that arose at high frequency by a mechanism not involving loss of CAT were not studied further. In the second method of mutant isolation, 7 I 50 survivors of u.v. treatment yielded no presumptive CAT ${ }^{-}$mutants.

Tests of reversion to chloramphenicol resistance were done on a serine-requiring mutant (strain 2522) of strain 2498. Spore suspensions treated with NTG yielded colonies at a frequency of about $10^{-6}$ on $10 \mu \mathrm{g}$ chloramphenicol $\mathrm{ml}^{-1}$. Two such putative revertants, which retained the proline and serine markers, were shown by the autoradiographic method to be $\mathrm{CAT}^{+}$.

\section{Genetic analysis of the CAT- mutation}

Recombination in S. acrimycini. Recombination was demonstrated in crosses between double auxotrophic derivatives of strain 1201 . These four-factor crosses were analysed by the standard procedure of plating on four selective media and characterizing samples of colonies on each medium for the segregation of non-selected markers (Hopwood, 1972). Unambiguous evidence of recombination was provided by the isolation of genotypes combining auxotrophic markers from both parents. Recombinants occurred at rather constant frequencies of a little less than $10^{-6}$. In addition to haploid recombinants, heterokaryons occurred at frequencies representing 2 to $80 \%$ of the total colonies arising on selective media, depending on the markers used for counter-selection. They were readily distinguished from haploid recombinants by their failure to be transferred by replica-plating to a medium of the same composition as that on which they originally developed, and by their giving rise exclusively to parental genotypes. This situation corresponded to that in $S$. rimosus 


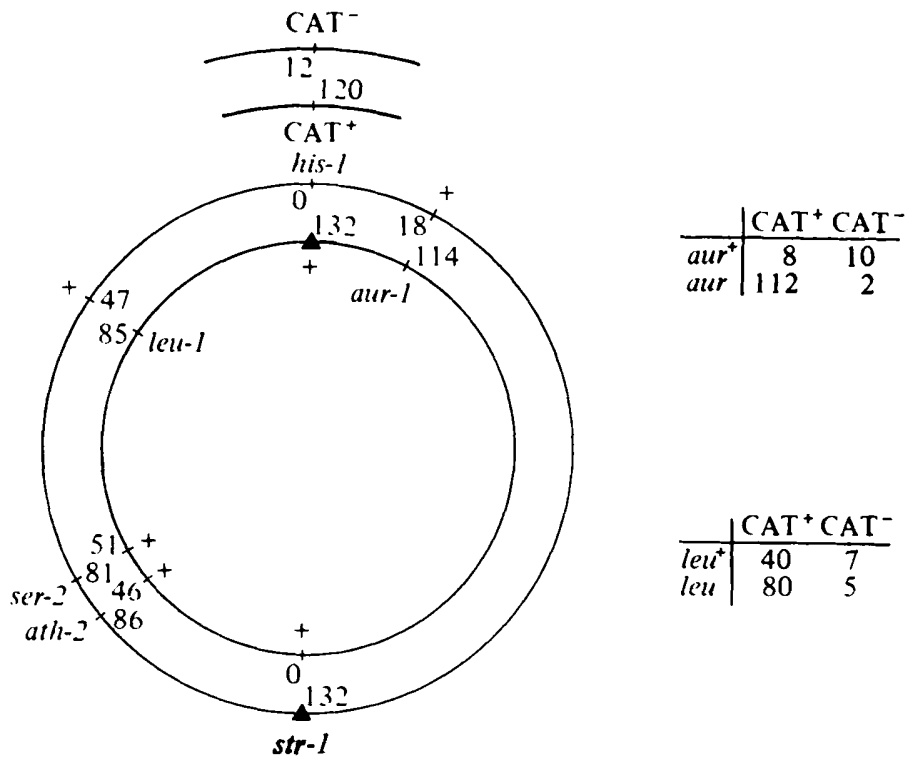

Fig. I. Results of a cross between $\mathrm{CAT}^{-}$strain 2564 (outer circle) and $\mathrm{CAT}^{+}$strain 2326 (inner circle). Recombinants inheriting his- $I^{+}$and $s t r-I$ (indicated by triangles) were selected and classified for the non-selected markers. Allele ratios indicate possible map positions for CAT between his-r and aur-I or between his-I and leu-I. Tests of independence between the segregation of CAT ${ }^{+1-}$ and either aur or leu indicate a position for CAT between his-I and aur-I: segregation of CAT $+1-$ is independent of that of leu $\left(\chi_{1}^{2}=2.97\right)$, but strongly dependent on that of aur $\left(\chi_{1}^{2}=54 \cdot 4\right)$. The individual genotype frequencies are in Table $\mathbf{I}$.

Table I. Progeny of the cross shown in Fig. I

\begin{tabular}{|c|c|c|c|}
\hline \multicolumn{2}{|c|}{ CAT $^{+}$} & \multicolumn{2}{|c|}{ CAT $^{-}$} \\
\hline Genotype* & Number & Genotype* & Number \\
\hline aur leu ser ath str & 37 & ser ath str & 4 \\
\hline aur leu str & 31 & leu ser ath str & 3 \\
\hline aur ser ath str & 27 & aur ser ath str & 2 \\
\hline aur leu ath str & 7 & leu str & 2 \\
\hline aur str & 5 & str & $\mathbf{I}$ \\
\hline leu ser ath str & 2 & & \\
\hline aur ser str & 2 & & \\
\hline aur ath str & 2 & & \\
\hline str & 2 & & \\
\hline aur leu ser str & I & & \\
\hline ser ath str & I & & \\
\hline leu ath str & $\mathbf{I}$ & & \\
\hline leu ser str & I & & \\
\hline ser str & $\mathbf{I}$ & & \\
\hline
\end{tabular}

(Friend \& Hopwood, 1971) and S. glaucescens (Baumann, Hütter \& Hopwood, 1974). When one parent in a cross carried a mutation conferring streptomycin resistance, heterokaryons were largely abolished on selective media containing streptomycin, as expected since streptomycin resistance is usually recessive.

Analysis of the patterns of marker segregation amongst haploid recombinants allowed the construction of a preliminary circular linkage map carrying six markers.

Crosses involving the $C_{A T}^{-}$mutation. In the first crosses between $\mathrm{CAT}^{+}$and $\mathrm{CAT}^{-}$ strains, four heterokaryons were allowed to segregate the two parental genotypes and 
samples of several hundred of each were tested for the CAT phenotype. There was no reassortment of CAT phenotype with chromosomal genotype, even when the genotype corresponding to the $\mathrm{CAT}^{+}$parent represented the majority of segregants from a particular heterokaryon. This result suggested chromosomal rather than plasmid linkage of the CAT ${ }^{-}$ mutation. However, it must be remembered that little is known about the behaviour of plasmids in Streptomyces heterokaryons. In S. scabies the finding that nearly all the segregants from heterokaryons between melanin-positive and melanin-negative strains were melanin-positive provided presumptive evidence for plasmid determination of melanin production (Gregory \& Huang, 1964), but unfortunately this remains the only evidence for such a plasmid in their strain.

More direct evidence for chromosomal linkage of the CAT- mutation came from the analysis of haploid recombinants selected from crosses between $\mathrm{CAT}^{+}$and $\mathrm{CAT}^{-}$strains. There was invariably a segregation of $\mathrm{CAT}^{+}$versus $\mathrm{CAT}^{-}$phenotype. The results of several crosses, in which the $\mathrm{CAT}^{-}$mutation was studied in various combinations with other markers, were completely compatible with a position for the CAT locus between his-I and aur-I (arginine plus uracil) on the circular chromosomal linkage group. An example of such a cross is shown in Fig. I and Table $I$.

\section{DISCUSSION}

The evidence that CAT in $S$. acrimycini IPVI610 is coded by a chromosomal gene is very strong. Variants lacking the activity did not arise with a high frequency such as might have reflected plasmid loss (only one, revertible, mutant occurred out of 8230 survivors of mutagenesis); there was no re-assortment of CAT phenotype with chromosomal genotypes in heterokaryons between $\mathrm{CAT}^{+}$and $\mathrm{CAT}^{-}$strains; and segregation of the $\mathrm{CAT}^{-}$phenotype amongst haploid recombinants from such crosses was that expected for a mutation in a chromosomal gene, which was mapped to the chromosomal linkage group. Further wildtype strains would, of course, have to be studied genetically in order to generalize this conclusion to other examples of antibiotic-inactivating enzymes in streptomycetes. In the strain of $S$. griseus studied by El-Kersch \& Plourde (1976b) some slight evidence for chromosomal determination of CAT was provided by the failure to induce CAT- variants by treatment with acriflavine or ethidium bromide.

A further interesting finding in the present study was that variants which were 10 -fold more sensitive than the starting strain, but still possessing CAT, arose with frequencies of 4 to $5 \%$ after u.v. or NTG treatment, and about I \% spontaneously. The induced frequency was two- to four-fold higher than that of total auxotrophs, so a general high mutability of the strain does not explain the results. These chloramphenicol-sensitive variants may correspond to those that occur in $S$. coelicolor A3(2), a strain that lacks CAT, in which they were shown not to arise by plasmid loss, but they nevertheless failed to map to the chromosome (Freeman et al., 1977). In S. acrimycini, genetic analysis was not refined sufficiently for a serious genetical investigation of these variants. The physiological basis of their chloramphenicol sensitivity is also unknown. It is interesting that, in $S$. acrimycini, the resistance shown by the wild type apparently depends on the simultaneous operation of two factors, a functional CAT enzyme and the wild-type state of the second, unknown, mechanism; in contrast, $S$. coelicolor achieves a higher resistance than that shown by $S$. acrimycini in the absence of CAT. 


\section{REFERENCES}

BaumanN, R., Hütter, R. \& Hopwood, D. A. (1974). Genetic analysis in a melanin-producing streptomycete, Streptomyces glaucescens. Journal of General Microbiology 81, 463-474.

Benveniste, R. \& Davies, J. (1973). Aminoglycoside antibiotic-inactivating enzymes in actinomycetes similar to those present in clinical isolates of antibiotic-resistant bacteria. Proceedings of the National Academy of Sciences of the United States of America 70, 2276-2280.

Courvalin, P., Weisblum, B. \& Davies, J. (1977). Aminoglycoside-modifying enzyme of an antibiotic-producing bacterium acts as a determinant of antibiotic resistance in Escherichia coli. Proceedings of the National Academy of Sciences of the United States of America 74, 999-1003.

Delić, V., Hopwood, D. A. \& Friend, E. J. (1970). Mutagenesis by $N$-methyl- $N$ '-nitro- $N$-nitrosoguanidine (NTG) in Streptomyces coelicolor. Mutation Research 9, 167-182.

El-KersCh, T. A. \& Plourde, J. R. (1976a). Biotransformation of antibiotics I. Acylation of chloramphenicol by spores of Streptomyces griseus isolated from the Egyptian soil. Journal of Antibiotics, Japan 24, 292-302.

El-Kersch, T. A. \& Plourde, J. R. (1976b). Biotransformation of antibiotics II. Investigation of the chloramphenicol acetyltransferase in Streptomyces griseus. Journal of Antibiotics, Japan 24, I 89-II98.

Freeman, R. F., Bibb, M. J. \& Hopwood, D. A. (1977). Chloramphenicol acetyltransferase-independent chloramphenicol resistance in Streptomyces coelicolor A3(2). Journal of General Microbiology 98, 453-465.
Friend, E. J. \& Hopwood, D. A. (1971). The linkage map of Streptomyces rimosus. Journal of General Microbiology 68, 187-197.

Gregory, K. F. \& HuANG, J. C. C. (1964). Tyrosinase inheritance in Streptomyces scabies. I. Genetic recombination. Journal of Bacteriology 87, I 28 I-I 286.

HaRold, R. J. \& Hopwood, D. A. (1970). Ultraviolet-sensitive mutants of Streptomyces coelicolor. I. Phenotypic characterization. Mutation Research 10, 427-438.

HOPWOOD, D. A. (1967). Genetic analysis and genome structure in Streptomyces coelicolor. Bacteriological Review's 31, 373-403.

HopwOOD, D. A. (1972). Genetic analysis in microorganisms. Methods in Microbiology 7 B, 29-158.

Hopwood, D. A., Chater, K. F., Dowding, J.E. \& Vivian, A. (1973). Advances in Streptomyces coelicolor genetics. Bacteriological Reviews 37, 371-405.

Nakano, H., Matsuhashi, Y., Takeuchi, T. \& UMEZAWA, H. (1977). Distribution of chloramphenicol acetyltransferase and chloramphenicol3-acetate esterase among Streptomyces and Corynebacterium. Journal of Antibiotics, Japan 30, 76-82.

Shaw, W. V. \& Hopwood, D. A. (1976). Chloramphenicol acetylation in Streptomyces. Journal of General Microbiology 94, 159-166.

ZaIDENZAIG, Y. \& SHAW, W. V. (1977). Characterization and comparison of chloramphenicol acetyltransferase variants. European Journal of Biochemistry (in the Press). 